\title{
AS RELAÇÕES ENTRE A ORIENTAÇÃO PARA O MERCADO, A INOVAÇÃ̃O DE PRODUTO E PROCESSO, E A PERFORMANCE ORGANIZACIONAL
}

\author{
THE RELATIONS BETWEEN MARKET GUIDANCE, PRODUCT \\ AND PROCESS INNOVATION, AND ORGANIZATIONAL \\ PERFORMANCE
}

\author{
RELACIONES DE LA ORIENTACIÓN AL MERCADO, UNA \\ INNOVACIÓN DE PRODUCTO Y PROCESO, Y RENDIMIENTO \\ DE LA ORGANIZACIÓN
}

\author{
Suelen Corrêa \\ Universidade de Passo Fundo (UPF), Brasil \\ sucorrea83@hotmail.com \\ Julio Cesar Ferro de Guimarães \\ Universidade Federal de Pelotas (UFPEL), Centro de \\ Engenharias (CEng), Brasil \\ juliocfguimaraes@yahoo.com.br
}

\section{Eliana Andréa Severo}

Universidade Potiguar (UNP), Programa de Pós-

Graduação em Administração (PPGA), Brasil

elianasevero2@hotmail.com

\author{
Márcia Rohr da Cruz \\ Universidade de Caxias do Sul (UCS), Brasil \\ marciarohrcruz@gmail.com
}

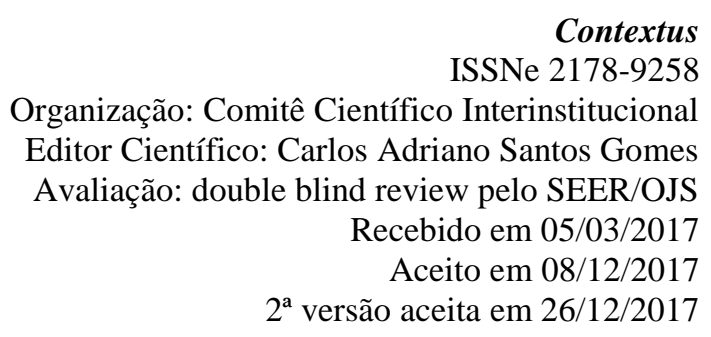

Contextus

ISSNe 2178-9258

Organização: Comitê Científico Interinstitucional Editor Científico: Carlos Adriano Santos Gomes

Recebido em 05/03/2017

Aceito em 08/12/2017

$2^{a}$ versão aceita em 26/12/2017

\section{RESUMO}

O alcance de uma performance organizacional superior é desejável para assegurar a permanência e o crescimento das empresas. Contudo, um ambiente de negócios marcado pelas rápidas mudanças tecnológicas e de mercado exige das empresas novas respostas ou a combinação de diferentes estratégias. O objetivo do presente estudo foi verificar a influência da orientação para o mercado sobre a inovação de produto e processo, bem como destes sobre a performance organizacional. Para isso, foi realizada uma survey, aplicada a gerentes e proprietários de empresas do estado do Rio Grande do Sul, e utilizada a técnica de Modelagem de Equações Estruturais para a análise dos dados. Foram encontrados relacionamentos positivos e significativos entre os construtos estudados, e observado que a inovação de processo contribui mais para a performance organizacional que a inovação de produto. Assim, administradores de empresas que busquem se manter e crescer no mercado devem investir mais nas inovações de processo que nas inovações de produto, já que elas contribuem para a redução de custo e aumento da qualidade dos produtos, proporcionando às empresas uma performance organizacional superior.

Palavras-chave: Orientação para o mercado. Inovação de produto. Inovação de processo. Performance organizacional.

\footnotetext{
ABSTRACT

To achieve a higher organizational performance is desirable to ensure the permanence and growth of companies. However, a business environment marked by rapid technological and market changes demands from companies new responses or a combination of different strategies. The purpose of this study was to verify the influence of market orientation on product and process innovation, as well as of these on organizational performance. For this,
} 
a survey was carried out, applied to managers and owners of companies in the state of Rio Grande do Sul, and the Structural Equations Modeling technique was used to analyze the data. We found positive and significant relationships among the constructs studied, and observed that process innovation contributes more to organizational performance than product innovation. Thus, business managers who seek to stay and grow on the market must invest more in process innovations than in product innovations, as they contribute to cost reduction and product quality enhancement, giving companies a superior organizational performance.

Keywords: Market orientation. Product Innovation. Process Innovation. Organizational Performance.

\section{RESUMEN}

El alcance de un desempeño organizacional superior es deseable para asegurar la permanencia y el crecimiento de las empresas. Sin embargo, un entorno de negocios marcado por los rápidos cambios tecnológicos y de mercado exige de las empresas nuevas respuestas o la combinación de diferentes estrategias. El objetivo del presente estudio fue verificar la influencia de la orientación hacia el mercado sobre la innovación de producto y proceso, así como de éstos sobre el desempeño organizacional. Para ello, se realizó una encuesta, aplicada a gerentes y propietarios de empresas del estado de Rio Grande do Sul, y utilizada la técnica de Modelado de Ecuaciones Estructurales para el análisis de los datos. Se han encontrado relaciones positivas y significativas entre los constructos estudiados, y observado que la innovación de proceso contribuye más al desempeño organizacional que la innovación de producto. Así, administradores de empresas que busquen mantenerse y crecer en el mercado deben invertir más en las innovaciones de proceso que en las innovaciones de producto, ya que ellas contribuyen a la reducción de costo y aumento de la calidad de los productos, proporcionando a las empresas un desempeño organizacional superior. Palabras clave: La orientación al mercado. La innovación del producto. Proceso de innovación. Rendimiento de la Organización.

\section{INTRODUÇÃO}

$\mathrm{O}$ alcance de uma performance organizacional superior é desejável para assegurar a permanência e o crescimento das empresas no mercado. Contudo, um ambiente de negócios mais acirrado e de rápidas mudanças requer das empresas a utilização de diferentes estratégias. Nesse contexto, a orientação para o mercado, bem como a inovação são algumas das estratégias que as empresas podem considerar, a fim de obter um melhor resultado organizacional.

$$
\text { Organizações orientadas para o }
$$
mercado buscam informações da clientela e as distribuem internamente por todos os setores, assim como lançam produtos e serviços alinhados às necessidades $\mathrm{e}$ expectativas dos clientes (KOHLI;
JAVORSKI, 1990). Evidências sugerem que tais empresas apresentam um desempenho superior (KOHLI; JAWORSKI, 1993; SLATER; NARVER, 1994; NARVER; SLATER; TIETJE, 1998).

Também a inovação tem sido utilizada para melhorar o desempenho empresarial, assegurando a sobrevivência e a competitividade (DAMANPOUR, 1991; PORTER, 1992). Kim e Maubourgne (2004) são ainda mais incisivos em suas afirmações; para eles, a inovação é a única alternativa de que as organizações dispõem para crescer e prosperar no mercado.

O presente estudo trata das relações, já sugeridas pela literatura, entre os temas: orientação para o mercado, inovação de produto e processo, e suas consequências sobre a performance organizacional. $\mathrm{O}$ 
estudo testa empiricamente esses elos, o que se alinha a pesquisas anteriores já com confirmações empíricas, sobretudo, da ligação entre orientação para o mercado e inovação de produto, bem como entre inovação de produto e performance organizacional.

Contudo, no que se refere à associação entre orientação para o mercado e inovação de processo, bem como entre inovação de processo e a performance organizacional, poucos estudos foram encontrados, nas bases de dados Scopus e Web of Science, que associam esses temas, especificamente, bem como os resultados desses relacionamentos. Portanto, decidiu-se testar empiricamente seus efeitos.

Logo, com base nessas evidências, formulou-se o objetivo do estudo, qual seja, testar o efeito da orientação para o mercado sobre duas formas de inovação - as inovações de produto e processo - e destes sobre a performance das organizações, utilizando para isso a técnica de Modelagem de Equações Estruturais.

\section{HIPÓTESES DA PESQUISA}

\subsection{Orientação para o mercado e inovação de produto e Processo}

Derivada do conceito de marketing, a orientação para o mercado consiste em uma preocupação das organizações em entender o consumidor, disseminar essas informações por toda a empresa, e responder adequadamente ao mercado, na oferta de produtos e serviços que correspondam às suas necessidades e expectativas (KOHLI; JAVORSKI, 1990). Devendo permear todos os setores da empresa, a orientação para o mercado deve constituir um elemento da cultura corporativa (HULT; HURLEY; KNIGHT, 2004).

O reconhecimento das necessidades atuais e futuras dos consumidores, bem como os fatores ambientais atuais e futuros (concorrentes, regulações do governo, tecnologia, entre outros) que possam influenciar essas preferências consiste na geração da inteligência de mercado (NARVER; SLATER, 1990). A observância dessas informações no lançamento das novas ofertas da empresa é chamada de responsividade, e resulta na criação coordenada de valor ao consumidor (KOHLI; JAVORSKI, 1990). É a cultura corporativa que vai expressar o nível de valor contínuo que a organização se dispõe a entregar a seus consumidores (NARVER; SLATER, 1994b; HAN; KIM; SRIVASTAVA, 1998).

Para Narver e Slater (1990) uma empresa orientada para o mercado é aquela que se encontra dirigida a seus consumidores e concorrentes; os autores trazem, ainda, um novo componente para o conceito de orientação para o mercado. Para eles, uma 
organização orientada para o mercado deve se ocupar da coordenação entre as várias funções da empresa.

Empresas orientadas ao mercado conseguem visualizar antecipadamente as necessidades dos clientes em desenvolvimento e responder a elas utilizando produtos e serviços inovativos, o que lhes dá uma vantagem de resposta às oportunidades e ameaças ambientais, além de um certo controle sobre o preço de seus produtos e serviços (NARVER; SLATER, 1995; HURLEY; HULT, 1998; NARVER; SLATER; TIETJE, 1998).

Logo, pode-se reconhecer a orientação para o mercado como um antecedente da inovação e do sucesso dos novos produtos (SLATER; NARVER, 1994b), bem como um antecedente para a capacidade de inovação (HURLEY; HULT, 1998). Para Kohli e Jaworski (1996), a orientação para o mercado é um antecedente para a inovação, e tem estado ausente nos modelos de orientação para o mercado.

Inovação é o resultado da atuação dos empreendedores que, ao oferecer ao mercado novas ofertas, causam uma ruptura no estado atual das coisas, o que Schumpeter (1934) chama de destruição criativa. Assim, novas combinações, como a introdução de um produto, ou mesmo a abertura de um novo mercado, a conquista de uma nova fonte de matéria-prima ou de bens semimanufaturados, e uma nova estruturação organizacional de uma indústria consistem em inovações (SCHUMPETER, 1934).

A introdução de um produto ou serviço, processo, método de marketing ou organizacional novo para a empresa consiste em uma inovação (OCDE, 2005). Assim, são consideradas inovações tanto aquelas novidades desenvolvidas pela própria empresa e, portanto, frutos de seu esforço, bem como àquelas introduzidas pela empresa no mercado, embora já conhecidas pelos consumidores e concorrentes (OCDE, 2005).

Especificamente, um produto novo e superior é aquele que: i) atende as necessidades do consumidor, oferecendo-lhe características únicas e maior qualidade, se comparados aos produtos da concorrência; ii) faz uma tarefa ao consumidor que outros produtos não conseguem executar; iii) consiste em um produto altamente inovativo e, portanto, novo para o mercado; iv) proporciona redução de custo para o consumidor (COOPER, 1980).

As definições de Paladino (2007) reforçam e vem ao encontro dos conceitos apresentados por Cooper (1980) para definir um novo produto. Segundo Paladino (2007), um novo produto é aquele que apresenta qualidade, funcionalidades e distintas aplicações se comparado à concorrência. Ademais, um novo produto é aquele construído sobre um amplo e consistente 
conhecimento tecnológico (PALADINO, 2007).

E, apesar da literatura enfatizar o forte relacionamento entre os construtos orientação para o mercado e inovação de produto (SLATER; NARVER, 1994b; ATUAHENE-GIMA, 1996; ATUAHENEGIMA; KO, 2001; LIU; SU, 2013), estudos empíricos têm demonstrado diferentes resultados. Ausência de relacionamento significativo entre os construtos orientação para o mercado e inovação de produto (LAWTON; PARASURAMAN, 1980), ou mesmo resultados limitados, que ora encontram relacionamentos significativos positivos (ATUAHENE-GIMA, 1996; GATIGNON; XUEREB, 1997; CARUNA; PITT; BERTHON, 1998) ora significativos negativos (ATUAHENE-GIMA, 1996), dependendo das variáveis analisadas, foram obtidos pelos autores em seus estudos empíricos.

No estudo executado por Lukas e Ferrel (2000) a orientação para o mercado apresenta um efeito sobre a inovação de produto. A orientação para o cliente aumenta a introdução de produtos novos para o mundo, e reduz o lançamento de produtos mee-too (novos para a organização mas não novos para o mercado); a orientação para o concorrente aumenta a introdução de produtos mee-too enquanto reduz o lançamento de extensões de linha e de novos produtos para o mundo e, por fim, a coordenação entre os setores da empresa aumenta o lançamento de extensões de linha, enquanto reduz a introdução de produtos mee-too (LUKAS; FERREL, 2000).

Ao verificar a influência da orientação para o mercado sobre pequenas empresas, Verhees e Meulenberg (2004) encontraram que a inteligência de mercado sobre o consumidor se relaciona positivamente com a inovação de produto, ao passo que a inteligência de mercado sobre o fornecedor ora funciona positiva ou negativamente. Assim, quando a empresa é menos inclinada a inovar, a inteligência de mercado sobre o fornecedor influencia positivamente a inovação de produto; porém, quando a empresa é muito forte em inovação, a inteligência sobre o mercado acaba por inibir a inovação de produto da empresa (VERHEES; MEULENBERG, 2004).

Por outro lado, para alguns autores uma forte orientação para o mercado pode conduzir a empresa a implementar imitações e produtos pouco inovativos (LAWTON; PARASURAMAN，1980; ATUAHENEGIMA, 1996), além de uma barreira para a comercialização de novas tecnologias e reduzida competitividade (CHRISTENSEN; BOWER, 1996). Os achados de Li, Lin e Chu (2008), em parte, vão ao encontro desses resultados.

Então, tendo como base o relacionamento positivo encontrado na 
literatura entre os construtos orientação para o mercado e a inovação de produto (GATIGNON; XUEREB, 1997; CARUNA; PITT; BERTHON, 1998; HAN; KIM; SRIVASTAVA, 1998; VERHEES; MEULENBERG, 2004; LIU; SU, 2013), a primeira hipótese da pesquisa é determinada:

\section{H1: A orientação para o mercado está positivamente relacionada com a inovação de produto.}

Estudos realizados durante os anos 1970 e 1980 atribuem o sucesso dos novos produtos e da inovação à orientação para o mercado (SLATER; NARVER, 1994b), o que é corroborado por Paladino (2007), que encontrou um resultado positivo e significativo entre o construto orientação para o mercado e a inovação.

E, ao passo que uma inovação de produto consiste em um arranjo criativo, específico e viável de características tecnológicas e de mercado (DOUGHERTY, 1992; MITCHEL, 1992), para que o novo produto alcance o sucesso, parece natural que suas características sejam determinadas mediante o conhecimento das expectativas e necessidades do mercado, observando-se a tecnologia empregada pela concorrência, ou seja, que a implementação deste novo produto seja uma tarefa posterior ao conhecimento deste mercado.
Apesar das inovações de produto acompanharem as inovações de processo, e das inovações de processo geralmente serem necessárias a partir da realização de inovações de produto, para que o conhecimento nesses dois tipos específicos de inovação aumente e se consolide, faz-se necessário examiná-las separadamente (BLAUG, 1963). Isso porque a habilidade da empresa em implementar inovações de produto e processo determina a sua competitividade

(DAMANPOUR, GOPALAKRISHNAN, 2001).

Um aperfeiçoamento técnico realizado sobre o processo de produção de um determinado bem, ainda não utilizado pela empresa, e que busca a redução do custo médio unitário do produto, constitui uma inovação de processo (BLAUG, 1963). Pode ser operacionalizada, portanto, por meio da introdução de uma nova máquina ao processo produtivo, ou mesmo através da reorganização do maquinário já utilizado pela fábrica (BLAUG, 1963).

O uso de novas técnicas, softwares ou procedimentos que alterem significativamente a forma pela qual a empresa produz ou distribui os seus produtos consiste em uma inovação de processo (OCDE, 2005). Geralmente, inovações de processo tem como objetivo a redução dos custos de produção ou distribuição, bem como a melhoria da qualidade, ou mesmo a produção de novos produtos (OCDE, 2005). 
Logo, partindo dos resultados empíricos positivos que relacionam a orientação para o mercado à inovação (HAN; KIM; SRIVASTAVA， 1998; PALADINO, 2007; LI; LIN; CHU, 2008), e dos estudos que associam a orientação para o mercado à inovação de produto (GATIGNON; XUEREB, 1997; CARUNA; PITT; BERTHON, 1998; HAN; KIM; SRIVASTAVA， 1998; VERHEES; MEULENBERG, 2004; LIU; SU, 2013), além das evidências de Blaug (1963), que sugere que as inovações de processo e produto se apresentam juntas, porém é necessário separá-las para um melhor entendimento, é delimitada a segunda hipótese da pesquisa:

\section{H2: A orientação para o mercado está} positivamente relacionada com a inovação de processo.

\subsection{Inovação de produto, processo e a performance organizacional}

A maioria da pesquisa sobre inovação tem avaliado o seu impacto sobre a performance (KOHLI; JAWORSLI, 1993). E, embora alguns estudos não apresentem influência da inovação sobre o desempenho organizacional, outras pesquisas tem obtido resultados positivos entre esse relacionamento (HAN; KIM, SRIVASTAVA，1998; LI; ATUAHENE-
GIMA， 2001;

ROSENBUSCH;

BRINCKMANN; BAUSCH, 2010).

O desempenho de uma organização consiste no resultado final da sua atuação no mercado, pressupondo uma complexa equação formada por uma série de variáveis, em interação (MARCH; SUTTON, 1997). Havendo interesse de pesquisadores e gerentes, um desempenho superior é desejável a organizações que busquem sobrevivência e crescimento.

O resultado organizacional é expresso em grande parte dos estudos por índices financeiros, como retorno sobre o investimento (ROI) (PALADINO, 2007; SOK; O'CASS, 2015), retorno sobre os ativos da empresa (ROA) (DAMANPOUR; GOPALAKRISHNAN, 2001; PALADINO, 2007; ROSENBUSCH; BRINCKMANN; BAUSCH，2010; SALVADÓ-AMORES; CASTRO; NAVAS-LÓPEZ， 2014) ou lucratividade média (BAKER; SINKULA, 2005; DELANEY; HUSELID, 1996; MELVILLE; KRAEMER; GURBAXANI, 2004; REINARTZ; KRAFFT; HOYER, 2004), embora indicadores não financeiros, como satisfação dos consumidores (DELANEY, HUSELID， 1996; BAKER; SINKULA， 2005) e colaboradores (BAKER; SINKULA, 2005) também sejam medidas utilizadas para refletir o desempenho organizacional. Ainda, medidas intermediárias e finais de performance organizacional podem ser 
usadas (BAKER; SINKULA, 2005; PALADINO, 2007).

A média da satisfação dos consumidores e dos empregados, a retenção dos consumidores, os serviços prestados ao consumidor, além das percepções sobre a qualidade dos produtos, consistem em medidas intermediárias de performance (BAKER; SINKULA, 2005). Por outro lado, a média da lucratividade, vendas, o crescimento das vendas, o retorno sobre o investimento (ROI), o sucesso dos novos produtos e a fatia de mercado da empresa representam medidas agregadas finais de performance (BAKER; SINKULA, 2005). Logo, as medidas de performance excedem os indicadores financeiros, e podem ser tão boas ou superiores a eles (BAKER; SINKULA, 2005; PALADINO, 2007).

Para a determinação da performance organizacional, Reinartz, Krafft e Hoyer (2004) combinaram medidas objetivas e subjetivas de desempenho, estratégia também utilizada por Damanpour e Gopalakrishnan (2001) para avaliar a influência das inovações de produto e processo sobre a performance organizacional no setor bancário. E Paladino (2007), por sua vez, utiliza medidas subjetivas de desempenho, bem como indicadores intermediários e totais, para determinar a performance organizacional.

Ao estudar a relação entre as despesas em P\&D e os resultados da inovação, expressos pelo número de patentes e pelos novos produtos da empresa, foi encontrado um relacionamento positivo entre inovação de produto e performance organizacional. Os dados confirmam, ainda, a afirmação de que, embora o sucesso de um novo produto aumente o seu lucro individual, é a capacidade da empresa de continuamente ofertar inovações ao mercado que possibilitará o alcance de um nível de lucratividade maior pela empresa como um todo (ARTZ et al., 2010).

Logo, baseado no relacionamento positivo existente entre a inovação de produto e a performance organizacional (ARTZ et al., 2010; LIN; TAM; GENG, 2013; SALVADÓ-AMORES; CASTRO; NAVAS-LÓPEZ, 2014; SOK; O’CASS, 2015), apresenta-se a terceira hipótese da pesquisa:

\section{H3: A inovação de produto está positivamente relacionada com a performance organizacional.}

As estratégias de novos produtos escolhidas pela empresa, bem como a implementação de inovações técnicas (de produto e/ou serviço e processo) apresentam um efeito sobre a performance organizacional (COOPER, 1984; HAN; KIM; SRIVASTAVA, 1998).

Schroeder (1990), ao estudar o impacto da introdução de uma nova 
tecnologia no processo produtivo de cinco fábricas de fundição observou, ao longo de 25 anos, as consequências que essa inovação causou para a própria empresa e para as demais empresas do mesmo grupo estratégico. A implementação da inovação, bem como o uso de tecnologias complementares e o próprio conhecimento que o seu uso contínuo proporcionou, permitiram à empresa uma melhor posição competitiva diante das demais.

$\mathrm{E}$, assim como estudos anteriores tem verificado a existência de relações positivas entre a inovação de produto e a performance organizacional (ARTZ et al., 2010;
SALVADÓ-AMORES; CASTRO; LIN; TAM; GENG, 2013; NAVAS-LÓPEZ, 2014; SOK; O’CASS, 2015), e as inovações de produto e processo aparecerem, em grande parte, interligadas, sendo necessária a sua separação para um melhor entendimento (BLAUG, 1963), sugere-se a quarta hipótese da pesquisa:

H4: A inovação de processo está positivamente relacionada com a performance organizacional.

As hipóteses testadas pelo estudo são apresentadas na Figura 1.

Figura 1 - Modelo e hipóteses da pesquisa

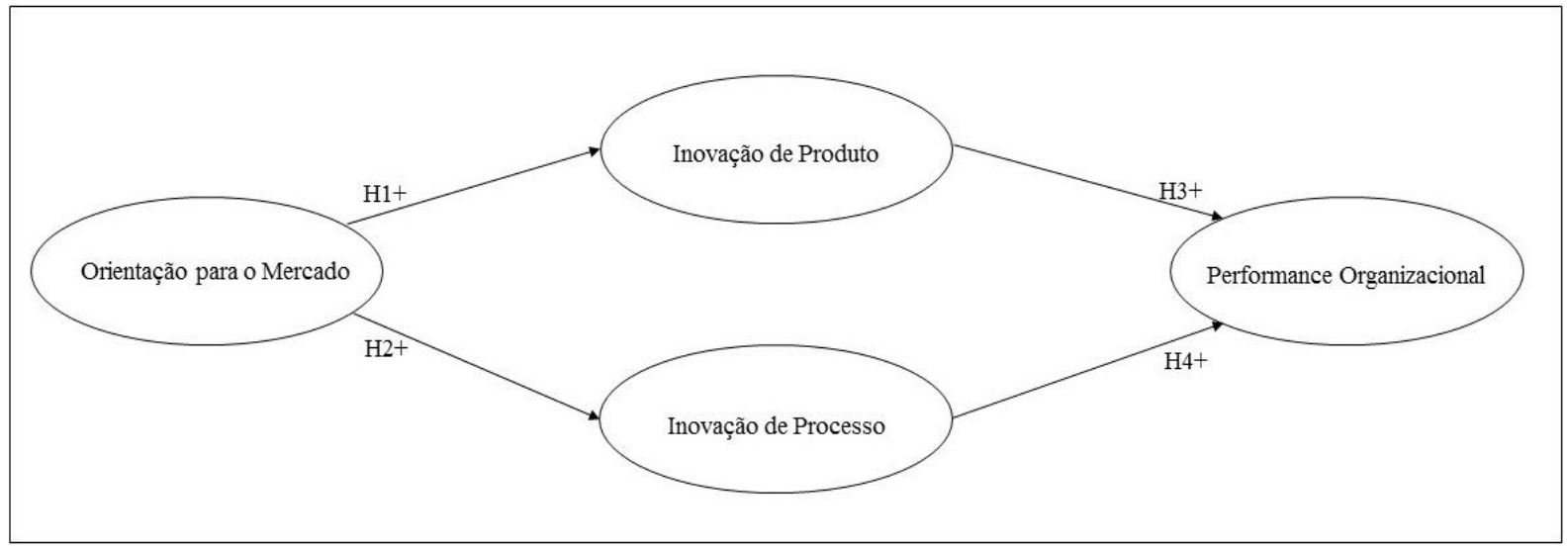

Fonte: elaboração própria (2016).

\section{MÉTODO}

A pesquisa realizada classifica-se como quantitativa e descritiva. Uma pesquisa quantitativa obtém seus dados em forma de números, geralmente se utiliza de uma amostra grande e representativa da população geral que se está estudando e envolve algum tipo de análise estatística (MALHOTRA, 2011). Ademais, mensura variáveis previamente determinadas, além da influência de uma variável sobre as outras; busca generalizações (APPOLINÁRIO, 2006). 
A pesquisa descritiva, por sua vez, visa medir características, descrever um determinado fenômeno ou verificar a associação de certos eventos (HAIR Jr. et al., 2005; MALHOTRA, 2011; ROESCH, 2013). É orientada por hipóteses, que serão testadas, e geralmente utiliza um questionário com número determinado de opções. Utilizada quando existe um sólido conhecimento do assunto, a pesquisa descritiva, com frequência, é confirmatória (HAIR Jr. et al., 2005; MALHOTRA, 2011).

A população investigada para verificar os efeitos da orientação para o mercado sobre a inovação de produto, de processo e destes sobre a performance organizacional, consiste em empresas do estado do Rio Grande do Sul. A amostra da pesquisa, por sua vez, consiste em 341 empresas. Caracteriza, portanto, uma amostra não-probabilística, e utiliza os critérios de acesso e conveniência na seleção das empresas respondentes (MALHOTRA, 2011).

O número de empresas respondentes atende às determinações de Hair Jr. et al. (2010), que sugerem o número de 15 entrevistados para cada variável a ser utilizada, além de um número entre 100 e 400 respondentes, e as sugestões de Kline (2011), que sugere um número mínimo de 200 respondentes para uma análise SEM.

Os dados necessários a este estudo foram coletados através de uma survey, por meio de um questionário, enviado por e-mail ou respondido através de contato pessoal. A elaboração do questionário de pesquisa foi feita de acordo com o modelo teórico apresentado na Tabela 1.

Cada variável foi classificada pelos respondentes por meio de uma escala Likert de 5 pontos, consistente com Paladino (2007), onde o número 1 indica discordo totalmente, o número 2, discordo parcialmente, o número 3 , nem concordo nem discordo, o número 4 expressa a concordância parcial com a afirmação apresentada e, por fim, o número 5, que significa a concordância total com a afirmativa feita.

A utilização de medidas subjetivas para avaliar a performance organizacional é consistente com estudos prévios que utilizaram a performance como variável dependente (DELANEY; HUSELID, 1996; REINARTZ, KRAFFT; HOYER, 2004; PALADINO, 2007). Ainda, pesquisas encontraram relações positivas, moderadas e fortes, entre medidas objetivas e perceptivas de desempenho, indicando a sua pertinência em refletir os índices verdadeiros de performance (POWELL, 1992).

A análise e interpretação dos dados obtidos com a pesquisa foi feita utilizandose a técnica de Modelagem de Equações Estruturais (SEM - Structural Equation Modeling). Para a tabulação e organização dos dados, foi utilizado o software Excel 
para Windows®. Após, para a realização dos testes estatísticos e análise dos dados, foi utilizado o software SPSS ${ }^{\circ}$ (Statistical
Package for Social Sciences), versão 21 para Windows ${ }^{\circledR}$ e, ainda, o software AMOS $®$ versão 21.

Tabela 1 - Variáveis e definições do questionário

\begin{tabular}{ll}
\hline Construto & Definições \\
\hline Orientação para o Mercado (OM) & "Orientação para o Mercado" (OM) consiste nos \\
& comportamentos e atitudes referentes à produção, \\
& distribuição e capacidade de retorno da empresa às \\
& informações obtidas com a inteligência de mercado. \\
& Elemento da cultura corporativa, representa o nível de \\
& preocupação da empresa em entregar valor contínuo ao \\
& consumidor. Essas afirmações teóricas baseiam-se em \\
Inovação de Produto (IP) & Kohli e Jaworski (1993), Hult, Hurley e Knight (2004). \\
& A implementação de um produto novo ou bastante \\
& aperfeiçoado, no que se refere a suas características ou \\
& mesmo usos diferentes, se comparados aos produtos \\
anteriores da empresa consiste na "Inovação de Produto" & (IP). Essas afirmações teóricas baseiam-se em OECD \\
Inovação de Processo (IPS) & (2005). \\
"Inovação de Processo" (IPS) é a utilização de novos & métodos ou métodos substancialmente alterados, na \\
fabricação ou distribuição de bens e serviços. Essas & afirmações são baseadas em OECD (2005). \\
Os resultados obtidos pela empresa no mercado em \\
relação a seus concorrentes consiste na sua "Performance \\
Organizacional" (PO). Aqui, estão relacionados aos \\
índices financeiros. Essas afirmações são baseadas em \\
Paladino (2007).
\end{tabular}

Fonte: elaboração própria (2016).

Então, procedeu-se à realização da técnica de Modelagem de Equações Estruturais (SEM), que consiste na Análise Fatorial Exploratória (AFE) e na Análise Fatorial Confirmatória (AFC), para verificar a alocação das variáveis nos construtos. Foram calculados o teste de esfericidade de Bartlett e a medida de adequação de Kayser, Meyer e Olkin (KMO), para avaliar a viabilidade da $\mathrm{AFE}$, bem como o Alpha de Cronbach, com o objetivo de verificar a confiabilidade das variáveis observáveis. Foi avaliado também o índice de Curtose, através do coeficiente de Mardia, visando verificar a normalidade dos dados (MARDIA, 1970), bem como analisada a assimetria dos dados (KLINE, 1998).

A AFE agrupou as variáveis em 4 fatores que, em sua maioria, mostraram-se superiores a 0,5 (HAIR Jr. et al., 2005). Apenas a variável IPS4 apresentou valor de 0,439 , portanto, abaixo do valor indicado de 0,5. Contudo, apesar da variável IPS4 apresentar uma carga fatorial um pouco mais baixa, foi decidido mantê-la no modelo, tendo em vista a sua contribuição na explicação do modelo. 
As comunalidades foram calculadas, que refletem o grau com que a solução de todos os fatores explica a variabilidade de uma determinada variável (HAIR Jr. et al.,

0,5, sendo decidido mantê-la no modelo, tendo em vista a sua importância
2005). Para Hair Jr. et al. (2005), comunalidades abaixo de 0,5 devem ser eliminadas. Nota-se que apenas a variável IPS4 apresentou comunalidade inferior a dentre as outras variáveis para explicar o fator.

Tabela 2 - Variáveis observáveis e latentes

\begin{tabular}{|c|c|c|c|}
\hline $\begin{array}{c}\text { Fator/Variável } \\
\text { Latente }\end{array}$ & Variáveis observáveis & Cargas fatoriais & Comunalidades \\
\hline \multirow{6}{*}{$\begin{array}{c}\text { Fator } \mathbf{1} \\
\text { Orientação } \\
\text { para o mercado }\end{array}$} & $\begin{array}{l}\text { OM1) Na empresa existe uma cultura corporativa, } \\
\text { caracterizada por uma disposição para fornecer valor } \\
\text { superior aos clientes de forma contínua. }\end{array}$ & 0,824 & 0,725 \\
\hline & $\begin{array}{l}\text { OM2) A empresa considera fundamentais as ações de } \\
\text { respostas às informações de demandas do mercado. }\end{array}$ & 0,815 & 0,728 \\
\hline & $\begin{array}{l}\text { OM3) A empresa considera prioritários os projetos e } \\
\text { ações de respostas às informações de tendências do } \\
\text { mercado. }\end{array}$ & 0,820 & 0,740 \\
\hline & $\begin{array}{l}\text { OM4) A empresa possui departamentos ou pessoas para } \\
\text { coletar e tratar informações do mercado, transformando- } \\
\text { as em inteligência de mercado. }\end{array}$ & 0,798 & 0,700 \\
\hline & $\begin{array}{l}\text { OM5) A empresa incorpora as informações de } \\
\text { inteligência de mercado ao processo de tomada de } \\
\text { decisões estratégicas. }\end{array}$ & 0,675 & 0,611 \\
\hline & $\begin{array}{l}\text { OM6) As inovações de Produto e Processo incorporam } \\
\text { as informações de inteligência de mercado. }\end{array}$ & 0,687 & 0,593 \\
\hline \multicolumn{2}{|c|}{ Média 4,131 } & \multicolumn{2}{|c|}{ Confiabilidade Composta 0,941 } \\
\hline \multirow{4}{*}{$\begin{array}{l}\text { Fator } 2 \\
\text { Inovação de } \\
\text { Produto }\end{array}$} & $\begin{array}{l}\text { IP1) A qualidade de nossos novos produtos é superior à } \\
\text { dos nossos concorrentes. }\end{array}$ & 0,734 & 0,617 \\
\hline & $\begin{array}{l}\text { IP2) Nosso produto, em termos de funcionalidades e } \\
\text { recursos, é superior em comparação com nossos } \\
\text { concorrentes. }\end{array}$ & 0,624 & 0,553 \\
\hline & $\begin{array}{l}\text { IP3) Nossos novos produtos incorporam um grande } \\
\text { corpo de novos conhecimentos tecnológicos. }\end{array}$ & 0,701 & 0,554 \\
\hline & $\begin{array}{l}\text { IP4) As aplicações de nossos novos produtos são } \\
\text { totalmente diferentes das aplicações dos produtos dos } \\
\text { nossos principais concorrentes. }\end{array}$ & 0,853 & 0,795 \\
\hline \multicolumn{4}{|c|}{ Média 3,859 } \\
\hline \multirow{4}{*}{$\begin{array}{l}\text { Fator } 3 \\
\text { Inovação de } \\
\text { Processo }\end{array}$} & $\begin{array}{l}\text { IPS1) As inovações de Processo aumentaram a } \\
\text { capacidade de produção ou de prestação de serviços. }\end{array}$ & 0,722 & 0,615 \\
\hline & $\begin{array}{l}\text { IPS2) As inovações de Processo aumentaram a } \\
\text { flexibilidade da produção ou da prestação de serviços. }\end{array}$ & 0,772 & 0,675 \\
\hline & $\begin{array}{l}\text { IPS3) As inovações de Processo reduziram os custos de } \\
\text { produção ou dos serviços prestados. }\end{array}$ & 0,741 & 0,558 \\
\hline & $\begin{array}{l}\text { IPS4) As inovações de processo melhoraram a qualidade } \\
\text { do produto. }\end{array}$ & 0,439 & 0,439 \\
\hline Média 4, & 124 Desvio-padrão 0,886 Alfa de Cronbach 0,722 Co & nfiabilidade Compc & osta 0,814 \\
\hline
\end{tabular}


(CONTINUAÇÃO)

PO1) O retorno sobre o investimento da nossa empresa é superior em relação aos nossos concorrentes.

0,833

0,776

PO2) O retorno sobre os ativos da nossa empresa é

0,772

0,799

Fator 4

superior em relação aos nossos concorrentes.

Performance

Organizacional

PO3) O total dos custos operacionais da nossa empresa é

menor aos custos totais de nossos concorrentes.

0,721

0,581

PO4) O desempenho global da nossa empresa no ano anterior foi superior aos principais concorrentes.

0,711

0,690

Média 3,307 Desvio-padrão 0,989 Alfa de Cronbach 0,848 Confiabilidade Composta 0,913

Fonte: dados da pesquisa (2016).

A Variância Média Extraída (VME) também foi calculada, para verificar a variância total de cada variável observável, e para avaliar o construto (FORNELL; LARCKER, 1982), sendo indicado o valor mínimo de 0,7 (HAIR Jr. et al., 2005), além de calculada a Variância Compartilhada (VC). Com o objetivo de verificar a associação existente entre duas variáveis (HAIR Jr. et al., 2005), seu valor deve ficar abaixo daquele obtido com a VME. Foram calculados, ainda, os índices Confiabilidade Composta de cada construto, bem como o indicador Confiabilidade Composta de todos os construtos, devendo resultar em valores superiores a 0,7 (MAROCO, 2010).

Procedeu-se, então, à avaliação do modelo integrado teórico. Para a avaliação do modelo, foi verificada a significância estatística dos coeficientes estimados e padronizados (HAIR Jr. et al, 2010), além de calculados os índices de ajuste absolutos e incrementais (HAIR Jr. et al., 2010).

Inicialmente, foram analisados os indicadores de ajuste absolutos, que avaliam a aproximação do modelo proposto pelo pesquisador à teoria (KLINE, 2011). Então, foram observados os resultados do Chiquadrado e dos Graus de Liberdade. Dividindo-se o Chi-quadrado pelos Graus de Liberdade, considera-se adequado um valor menor ou igual a 5 (TANAKA, 1993).

Foram calculados, ainda, os índices de ajuste absolutos GFI, RMSEA e RMR. O GFI (índice de qualidade do ajuste) (HAIR Jr. et al., 2010) demonstra a proporção que o modelo explica as covariâncias observadas (KLINE, 2011). Variando entre 0 e 1, quanto maior o valor, melhor é o ajuste do modelo (HAIR Jr. et al., 2007; KLINE, 2011). E, embora no passado tenha-se utilizado como uma boa medida de GFI o índice de 0.90 (HAIR Jr. et al, 2007), autores argumentam que deve ser buscado o valor de 0.95 para o GFI (HAIR Jr. et al, 2010).

Os valores indicados para o RMSEA (raiz quadrada média do erro de aproximação) devem variar entre 0,05 e 0,08 (KLINE, 2005; HAIR Jr. et al., 2007) e para o RMR (raiz quadrada média residual), 
valores mais próximos a zero são melhores (KLINE, 2011).

A seguir, foram calculados os índices de ajuste incrementais ou comparativos, que verificam o ajuste do modelo a um determinado modelo base, geralmente o modelo nulo (HAIR Jr. et al., 2010). O índice de adequação da normalidade (NFI) e o índice de ajuste comparativo (CFI) constituem índices de ajuste incrementais (HAIR Jr. et al., 2010).

Um dos mais conhecidos, o NFI deve estar entre 0 e 1 - quanto mais próximo o valor estiver de 1 , mais adequado é o modelo diante do modelo-base (HAIR Jr. et al., 2010). O CFI, outro índice de ajuste incremental, compara o ajuste do modelo do pesquisador a um modelo base, geralmente $o$ modelo independente, para verificar o de melhor ajuste (KLINE, 2011). Apresenta valores entre 0 e 1 , embora os valores desejáveis estejam acima de 0.90 .

\section{RESULTADOS}

Para verificar a relação da orientação para o mercado (OM) com a inovação de produto (IP) e Processo (IPS) e, destes sobre a performance organizacional (PO), foram aplicados 353 questionários a gerentes ou proprietários de empresas, pessoalmente ou por e-mail. A amostra da pesquisa caracteriza-se como não-probabilística, tendo os respondentes sido selecionados de acordo com o critério de facilidade de acesso e conveniência (MALHOTRA, 2011).

Como os respondentes foram previamente contatados e convidados a participar da pesquisa, sendo enviado por email ou aplicado o instrumento somente àqueles que concordaram em participar, todos os questionários enviados foram respondidos. Os questionários foram aplicados no período entre 05 de fevereiro e 26 de julho de 2016.

Depois de organizar e tabular os dados no software Excel, procedeu-se à limpeza dos dados, cujo objetivo foi eliminar aqueles que se caracterizaram como outliers ou que apresentaram dados faltantes (missings), seguindo a orientação de Hair Jr. et al. (2010), que sugere eliminar questionários que apresentarem mais de $10 \%$ de ocorrência. Assim, dos 353 questionários respondidos 12 foram excluídos da amostra, por concentrarem respostas em uma única questão (9 questionários - outliers) e apresentarem dados faltantes (3 questionários - missings).

Após a limpeza dos dados, restaram 341 questionários, que consistiram na amostra do estudo, número que atende às determinações de Hair Jr. et al. (2010), que sugerem a utilização de um número entre 100 e 400 respondentes para uma análise SEM, e excedem as de Kline (2011), que sugere o uso de 200 respondentes. Ainda, o número de 341 questionários excede às 
determinações quanto ao número mínimo de respondentes por variável; enquanto os autores sugerem, respectivamente, o uso de 10 e 15 respostas por variável, o estudo utiliza 18,9 (HAIR Jr. et al., 2005; KLINE, 2011).

No que se refere ao setor de atuação das empresas, a maioria delas, $49,4 \%$ é do setor da indústria de transformação, enquanto empresas do comércio e do setor de serviços apresentam percentuais bem próximos, na casa dos 25\%. Quanto ao porte, na pesquisa predominam as microempresas $(43,8 \%)$, ao passo que as pequenas e médias empresas representam, ambas, 27,6\%; apenas uma pequena minoria da amostra é formada por grandes empresas $(7,4 \%)$.

Realizada a análise descritiva dos dados, seguiu-se à realização da técnica de Modelagem de Equações Estruturais (SEM), que consiste na realização da Análise Fatorial Exploratória (AFE) e a Análise Fatorial Confirmatória (AFC). A realização dessas técnicas tem como objetivo verificar se os dados obtidos com a amostra confirmam o que está afirmado na teoria, mais precisamente, suas relações e construtos.

Inicialmente, para analisar as variáveis de cada construto, e, portanto, proceder à realização da Análise Fatorial Exploratória (AFE), foi utilizada a rotação Varimax, o que resultou em uma matriz de 4 fatores, com $65,266 \%$ de explicação para a variabilidade dos dados, superior ao mínimo indicado de 60\% (HAIR Jr. et al., 2005).

Foram calculados, ainda, o Alpha de Cronbach para todos os fatores, que atingiu o valor de 0,905 , portanto, superior ao mínimo indicado de 0,7 (HAIR Jr. et al., 2007), a medida de adequação de Kaiser, Meyer e Olkin (KMO), que atingiu o valor de 0,874 , de acordo com o sugerido (PESTANA; GAGEIRO, 2005) e o teste de esfericidade de Bartlett, que também se mostrou significativo.

Ainda, foi avaliado o índice de Curtose, através do coeficiente de Mardia, que apresentou valores menores que 5, o que indica normalidade dos dados (MARDIA, 1970). Também foi analisada a assimetria dos dados das variáveis observadas; apresentando valores próximos a zero, o índice indica uma simetria moderada (KLINE, 1998).

E, quanto às médias das respostas para cada variável, os construtos orientação para o mercado, inovação de processo e Produto apresentam os maiores valores, respectivamente, com desvio-padrão em todas as variáveis abaixo de 0,9 , indicando grande concordância nas respostas e um baixo desvio-padrão (HAIR Jr. et al., 2005). A menor média das respostas foi encontrada no construto performance organizacional; este foi o construto que apresentou maior desvio-padrão $(0,989)$, embora ainda menor que 1. 
Os índices Confiabilidade Composta de cada construto (Tabela 2), bem como o indicador Confiabilidade Composta de todos os construtos (Tabela 5), ambos se mostraram superiores ao valor mínimo sugerido de 0,7 (MAROCO, 2010). Logo, considera-se que as variáveis observáveis são consistentes em suas mensurações.

No que se refere ao cálculo da Variância Média Extraída (VME) (Tabela 3), enquanto os construtos orientação para o mercado e performance organizacional se apresentaram superiores ao mínimo indicado de 0,7 (FORNELL; LARCKER, 1982), os construtos inovação de produto e processo apresentaram valores inferiores a 0,7. Logo, a Variância Extraída de todos os construtos ficou ligeiramente abaixo do valor de referência de 0,7 (Tabela 5), resultando no valor de 0,665 (HAIR Jr. et al., 2005).

Quanto à Variância Compartilhada (VC), ou seja, a associação que pode ser observada entre duas variáveis (HAIR Jr. et al., 2005), com exceção da correlação entre o construto inovação de processo e performance organizacional, todas as outras apresentaram valores inferiores àqueles obtidos com o cálculo da VME (Tabela 3), e, portanto, mostram-se adequadas.

Tabela 3 - Variância Média Extraída e Variância Compartilhada

\begin{tabular}{|c|c|c|c|c|}
\hline & $\begin{array}{c}\text { Orientação para o } \\
\text { mercado }\end{array}$ & $\begin{array}{c}\text { Inovação de } \\
\text { produto }\end{array}$ & $\begin{array}{c}\text { Inovação de } \\
\text { processo }\end{array}$ & $\begin{array}{c}\text { Performance } \\
\text { organizacional }\end{array}$ \\
\hline $\begin{array}{c}\text { Orientação para o } \\
\text { mercado }\end{array}$ & $0,726^{a}$ & & & \\
\hline $\begin{array}{l}\text { Inovação de } \\
\text { produto }\end{array}$ & $0,477^{b}$ & $0,634^{\mathrm{a}}$ & & \\
\hline $\begin{array}{c}\text { Inovação de } \\
\text { processo }\end{array}$ & $0,498^{b}$ & $0,511^{b}$ & $0,531^{\mathrm{a}}$ & \\
\hline $\begin{array}{c}\text { Performance } \\
\text { organizacional }\end{array}$ & $0,562^{b}$ & $0,579^{b}$ & $0,593^{b}$ & $0,730^{\mathrm{a}}$ \\
\hline
\end{tabular}

a Variância Média Extraída

b Variância Compartilhada

Fonte: dados da pesquisa do relatório do SPSS (2016).

A seguir, realizou-se a Correlação de Pearson, que evidenciou 2 correlações superiores ao nível máximo de 0,7 , o que caracteriza multicolinearidade (HAIR Jr. et al., 2005). São elas: PO2 <--> PO1 e OM2 <--> OM1. Há multicolinearidade quando mais de uma variável é utilizada para medir os mesmos aspectos que outra (HAIR Jr. et al., 2007). No entanto, como esses valores se mostraram dentro do mesmo construto, eles são aceitáveis, pois indicam a concordância das variáveis em medir o construto.

Após a validação das escalas e a avaliação entre os construtos realizou-se a 
análise do modelo integrado teórico, relações entre os construtos orientação para formado pelos modelos de mensuração e o mercado, inovação de produto, inovação estrutural (Figura 2), a fim de verificar as de processo e performance organizacional.

Figura 2 - Modelo integrado teórico

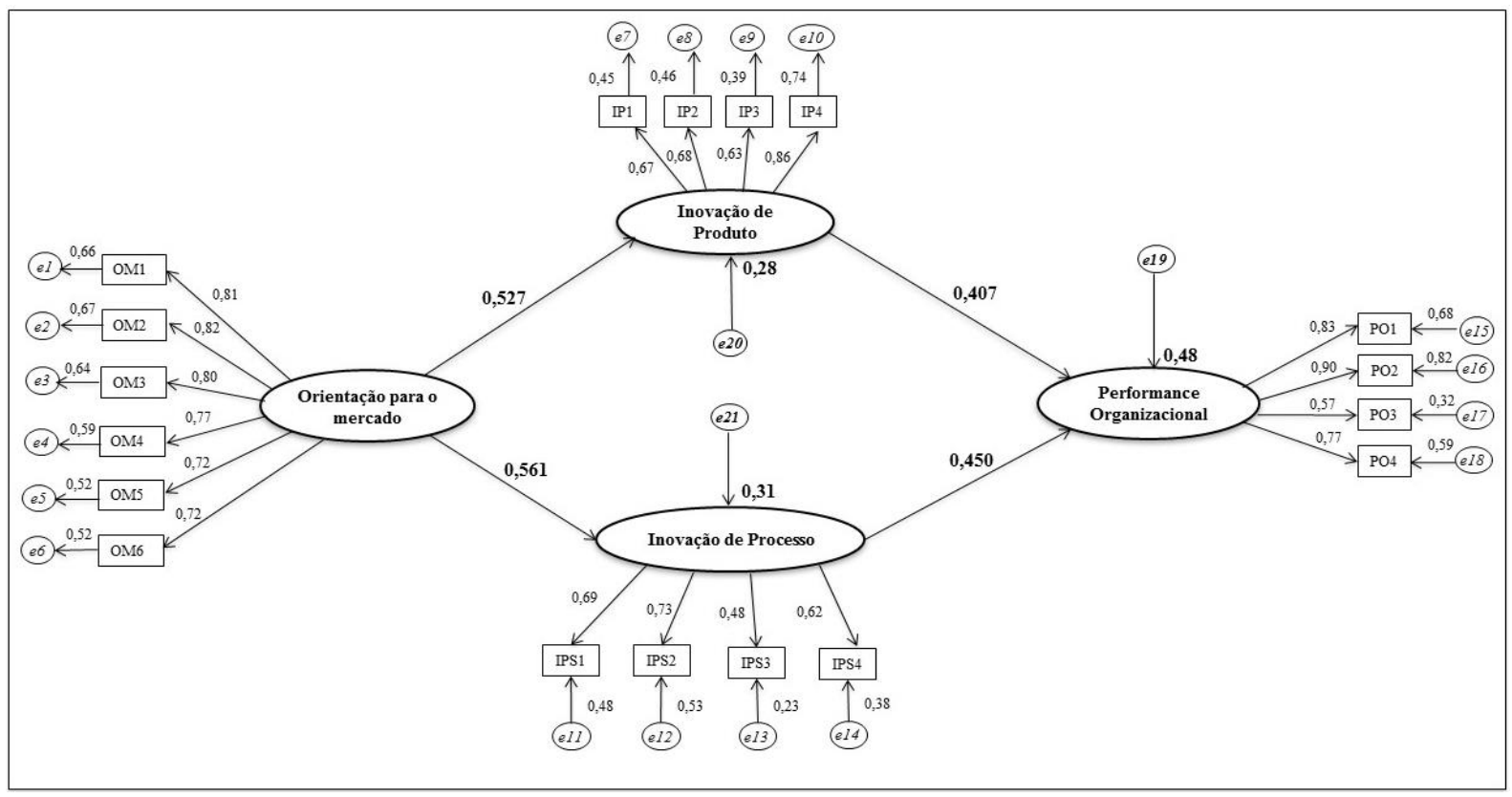

Fonte: dados da pesquisa (2016).

Procedeu-se, então, à avaliação do modelo integrado teórico, mediante o uso do software AMOS versão 21 acoplado ao SPSS® versão 21. Para a avaliação do modelo, foram verificadas a significância estatística dos coeficientes estimados e padronizados (Tabela 4), além de calculados os índices de ajuste absolutos e incrementais (HAIR Jr. et al., 2010) (Tabela 5).

As relações de dependência foram verificadas por meio dos testes de Hipótese de Correlação (EC) e Covariância (SC). Em ambos os testes, as hipóteses do estudo foram confirmadas, uma vez que todas as relações se mostraram positivas e significativas (Tabela 4). Também o índice Critical Ratio (CR) mostrou-se adequado, apresentando valores acima de $|1,96|$, o que indica que a estimativa é estatisticamente diferente de zero (BYRNE, 2010). 
Tabela 4 - Teste de Hipótese (Correlação e Covariância) - Modelo integrado teórico

\begin{tabular}{cccccccccc}
\hline Hipóteses & \multicolumn{2}{c}{ Construtos } & & $\begin{array}{c}\text { Estimate } \\
\text { Coefficient } \\
\text { (EC) }\end{array}$ & $\begin{array}{c}\text { Standardized } \\
\text { Coefficient } \\
\text { (SC) }\end{array}$ & $\begin{array}{c}\text { Standard } \\
\text { Deviation } \\
\text { (SD) }\end{array}$ & $\begin{array}{c}\text { Critical } \\
\text { ratio } \\
\text { (CR) }\end{array}$ & p \\
\hline H1 & PROD & $<---$ & MERC & 0,586 & 0,527 & 0,078 & 7,497 & $* * *$ \\
H2 & PROC & $<---$ & MERC & 0,523 & 0,561 & 0,072 & 7,253 & $* * *$ \\
H3 & PERFORM & $<---$ & PROD & 0,474 & 0,407 & 0,072 & 6,602 & $* * *$ \\
H4 & PERFORM & $<---$ & PROC & 0,624 & 0,450 & 0,095 & 6,551 & $* * *$ \\
\hline$* * *$ Significância $P<0,001$ & & & & & & & & &
\end{tabular}

Fonte: dados da pesquisa do relatório do Amos (2016).

Assim, as seguintes relações positivas entre os construtos foram confirmadas: i) orientação para o mercado e inovação de produto (Hipótese 1); ii) orientação para o mercado e inovação de processo (Hipótese 2); iii) inovação de produto e performance organizacional (Hipótese 3); iv) inovação de processo e performance organizacional (Hipótese 4).

Posteriormente, foram calculados e analisados os índices de ajuste absolutos e incrementais (Tabela 5). Inicialmente, foram analisados os indicadores de ajuste absolutos, aqueles que avaliam a aproximação do modelo proposto pelo

Tabela 5 - Índices de ajuste do modelo integrado pesquisador à teoria (KLINE, 2011). Assim, observou-se que o Chi-quadrado atinge o valor de 542,619, enquanto os Graus de Liberdade alcançam 131,000, a um nível de probabilidade de $\mathrm{p}<0,001$ (Tabela 5). A observação isolada desses índices sugere a existência de uma grande diferença entre as matrizes de covariância estimada e observada, o que poderia indicar a inadequação do modelo (HAIR Jr. et al., 2010). Porém, dividindo-se o Chi-quadrado pelo índice Graus de Liberdade, obtém-se o valor de 4,142, portanto, dentro do limite sugerido por Tanaka (1993), que é menor ou igual a 5 .

\begin{tabular}{lc}
\hline \multicolumn{1}{c}{$\quad$ Índice de ajuste } & Modelo integrado teórico \\
\hline Chi-quadrado & 542,619 \\
Graus de liberdade & 131,000 \\
Chi-quadrado dividido pelos graus de liberdade & 4,142 \\
Nível de probabilidade & $0,000^{*}$ \\
CFI - Comparative Fit Index & 0,870 \\
NFI - Normed Fit Index & 0,836 \\
GFI - Goodness of Fit Index & 0,851 \\
RMSEA - Root Mean Squared Error of Approximation & 0,096 \\
RMR - Root Mean Square Residual & 0,064 \\
KMO - Kaiser-Meyer-Olkin Measure of Sampling Adequacy & 0,874 \\
Variância Extraída & 0,665
\end{tabular}


(CONTINUAÇÃO)

Confiabilidade Composta

0,972

Alpha de Cronbach

0,905

* Significância $\mathrm{p}<0,001$

Fonte: elaboração própria (2016).

Foram calculados, ainda, o GFI, que se mostrou de acordo com o esperado (0,851) (HAIR Jr. et al., 2010), o RMSEA, que atingiu o valor de 0,096, um pouco acima do intervalo sugerido (HAIR Jr. et al.,

2010), mostraram-se muito próximos, embora abaixo dos valores indicados. Enquanto recomenda-se para ambos valores iguais ou maiores que 0,9 (HAIR Jr. et al., 2010), o modelo atingiu 0,870 para o CFI e 0,836 para o NFI.

$$
\text { Ainda, o } \mathrm{KMO}(0,874), \quad \text { a }
$$

Confiabilidade Composta $(0,972)$ e o Alpha de Cronbach $(0,905)$ apresentam valores acima dos recomendados (PESTANA; GAGEIRO, 2005; MAROCO, 2010; HAIR Jr. et al., 2007), ao passo que a Variância Extraída, cujo valor atingiu 0,665 encontrase ligeiramente abaixo do valor de referência, que é de 0,7 (HAIR Jr. et al., 1998).

Em suma, para determinar se um modelo é ou não adequado para representar a teoria, deve-se utilizar, além do $\mathrm{x}^{2}$, um indicador de ajuste absoluto e um índice de ajuste incremental (HAIR Jr. et al., 2010). E, embora $\mathrm{o} \mathrm{x}^{2}$ não demonstre um bom ajuste do modelo, uma vez que os resultados se
2007; KLINE, 2005) e o RMR, (0,064), portanto, adequado para KLINE (2011).

Os índices de ajuste incrementais ou comparativos, aqueles que avaliam o ajuste do modelo a um determinado modelo base, geralmente o modelo nulo (HAIR Jr. et al., mostram distintos aos esperados $\left(\mathrm{x}^{2}=\right.$ $542,619 \mathrm{p}<0,001$ ), os valores obtidos com a divisão do $\mathrm{x}^{2}$ com os Graus de Liberdade mostram-se satisfatórios $(4,142)$, bem como os valores obtidos com o cálculo do GFI $(0,851)$ e o $\operatorname{RMR}(0,064)$.

Ainda, os valores obtidos com o cálculo dos índices de ajuste incrementais mostram-se abaixo, embora muito próximos dos valores sugeridos $(\mathrm{CFI}=0,870$ e $\mathrm{NFI}=$ 0,836). Assim, os resultados obtidos pelos índices de qualidade de ajuste sugerem uma adequação do modelo à teoria utilizada. E, juntamente com a confirmação das relações de dependência, o estudo confirma a adequação do modelo proposto à realidade dos dados.

\section{DISCUSSÃO}

Os resultados mostram relações significativas entre todos os construtos estudados (Tabela 4). Assim, o construto orientação para o mercado $(\mathrm{OM})$ é um 
importante antecedente da inovação de produto (IP) e inovação de processo (IPS), e todos contribuem para a performance organizacional (PO).

Para a amostra investigada, a relação entre orientação para o mercado e Inovação $(\mathrm{SC}=0,527$ e $\mathrm{SC}=0,561)$ mostrou-se superior a relação entre Inovação e performance organizacional $(\mathrm{SC}=0,407 \mathrm{e}$ $\mathrm{SC}=0,450)$, o que sugere que a orientação para o mercado contribui mais para a Inovação (Produto e Processo), enquanto a Inovação apresenta uma influência menor sobre a performance organizacional.

O relacionamento entre OM ---> IP mostrou-se alto $(\mathrm{SC}=0,527)$, indicando que empresas orientadas para o mercado apresentam inovação de produto, vindo ao encontro de estudos anteriores (GATIGNON; XUEREB, 1997; CARUNA; PITT; BERTON, 1998; HAN; KIM; SRIVASTAVA, 1998; VERHEES; MEULENBERG, 2004; LIU; SU, 2013). A relação entre OM ---> IPS também foi positiva, o que corrobora com os resultados positivos e significativos encontrados entre a orientação para o mercado e a inovação (HAN; KIM; SRIVASTAVA， 1998; PALADINO, 2007; LI; LIN; CHU, 2008).

Contudo, o fato da relação entre OM ---> IPS ter sido positiva, significativa, e a mais alta encontrada no modelo $(\mathrm{SC}=$ 0,561), chama a atenção para esse relacionamento, e, logo, para a relevância da
OM na implementação das IPS, especificamente, e constitui um importante achado da pesquisa. Logo, estudos adicionais são necessários, a fim de confirmar esses efeitos. Entretanto, pode-se dizer que empresas orientadas para o mercado apresentam mais inovações de processo do que de produtos, embora ambas apresentem relações fortes.

Em se tratando da relação entre IP --> PO, o relacionamento mostrou-se positivo $(\mathrm{SC}=0,407)$, já evidenciado por estudos anteriores (ARTZ et al., 2010; LIN; TAM; GENG, 2013; SALVADÓ-AMORES; CASTRO; NAVAS-LÓPEZ，2014; SOK; O'CASS, 2015). E, quanto à relação entre IPS ---> PO, ela atingiu o valor de 0,450 (SC), convergindo para estudos anteriores que também evidenciaram relacionamentos positivos existentes entre a inovação e a performance organizacional (HAN; KIM, SRIVASTAVA, 1998; LI; ATUAHENEGIMA, 2001; ROSENBUSCH; BRINCKMANN; BAUSCH, 2010).

Ainda, de acordo com os dados obtidos com a pesquisa, pode-se afirmar que a IPS contribui mais para a PO que a IP, já que a relação entre IPS ---> PO $(\mathrm{SC}=0,450)$ mostrou-se superior à relação IP ---> PO (SC $=0,407)$, e consiste no segundo achado da pesquisa, tendo em vista que poucos estudos, nas bases de dados Scopus e Web of Science, analisaram o efeito da IPS sobre a PO. Logo, segundo os dados da pesquisa, as empresas 
que buscam uma PO superior devem focar seus esforços na implementação das IPS mais do que nas IP. Portanto, de acordo com o estudo, os construtos orientação para o mercado, inovação de produto e processo explicam $\quad 48 \%$ da performance organizacional de uma empresa.

Um resumo da avaliação das hipóteses testadas na pesquisa é apresentado na Tabela 6.

Tabela 6 - Hipóteses da pesquisa

\begin{tabular}{|c|c|c|}
\hline Hipóteses & Descrição & Confirmação \\
\hline $\mathrm{H} 1$ & $\begin{array}{l}\text { A orientação para o mercado está positivamente relacionada com a inovação de } \\
\text { produto }\end{array}$ & Confirmada \\
\hline $\mathrm{H} 2$ & $\begin{array}{l}\text { A orientação para o mercado está positivamente relacionada com a inovação de } \\
\text { processo }\end{array}$ & Confirmada \\
\hline H3 & $\begin{array}{l}\text { A inovação de produto está positivamente relacionada com a performance } \\
\text { organizacional }\end{array}$ & Confirmada \\
\hline H4 & $\begin{array}{l}\text { A inovação de processo está positivamente relacionada com a performance } \\
\text { organizacional }\end{array}$ & Confirmada \\
\hline
\end{tabular}

\section{CONSIDERAÇÕES FINAIS}

Analisar as relações existentes entre os construtos orientação para o mercado (KOHLI; JAWORSKI, 1993; HULT; HURLEY; KNIGHT, 2004), inovação de produto (PALADINO, 2007), inovação de processo (OCDE, 2005) e performance organizacional (PALADINO, 2007) consistiu no objetivo deste estudo. Para isso, foi conduzida uma survey com 341 empresas do estado do Rio Grande do Sul, cujos dados foram analisados mediante a técnica de Modelagem de Equações Estruturais.

A realização da pesquisa evidenciou a existência de relacionamentos positivos e significativos entre os construtos orientação para o mercado e inovação de produto $(\mathrm{SC}=$ $0,527)$ e de processo $(\mathrm{SC}=0,561)$ (Tabela 19), o que demonstra a importância do construto orientação para o mercado como antecedente dos construtos inovação de produto e de processo. Portanto, empresas que se apresentam próximas do seu mercado inovam em produtos e processos.

Também foram encontrados resultados positivos e significativos, embora menores, entre os construtos inovação de produto e performance organizacional $(\mathrm{SC}=$ 0,407) e inovação de processo e performance organizacional $(\mathrm{SC}=0,450)($ Tabela 18$)$, evidenciando esses construtos como importantes antecedentes da performance organizacional. Logo, organizações que concentram seus esforços na implementação das inovações de produto e processo atingem uma melhor performance organizacional.

$$
\mathrm{O} \text { relacionamento positivo e }
$$
significativo encontrado entre os construtos orientação para o mercado e inovação de 
processo, e inovação de processo e performance organizacional consistem em relevantes contribuições acadêmicas. Como a literatura que trata desses temas não trata dessas relações especificamente, sugere-se a realização de outros estudos que possam esclarecer ou fortalecê-los.

A validação estatística das escalas utilizadas no estudo, bem como a construção de um framework de pesquisa para analisar a influência dos construtos orientação para o mercado, inovação de produto, processo e a performance organizacional também constituem contribuições da pesquisa. Assim, acadêmicos que queiram investigar os mesmos construtos podem utilizar as escalas e o modelo aqui construído.

Orientação para o mercado, inovação de produto e processo contribuem para uma melhor performance organizacional. Contudo, de acordo com os dados obtidos com o estudo, é a implementação da estratégia de orientação para o mercado combinada à inovação de processo, mais que a inovação de produto, que produz uma melhor performance organizacional. Assim, administradores que buscam um desempenho superior das suas organizações precisam entender as necessidades e expectativas de seus clientes, bem como focar seus esforços em novos processos de produção e entrega dos produtos, o que proporciona redução de custo e aumento de qualidade e, como consequência, aumento da performance organizacional.

A realização do estudo evidencia também algumas limitações. O número utilizado para a amostra, embora suficiente para a técnica de coleta de dados utilizada, não é representativo da população estudada e, por isso, não possibilita a indução dos seus resultados. Além disso, os resultados obtidos são limitados para o tipo de empresa respondente, região e contexto socioeconômico - pesquisa semelhante realizada em outras regiões pode apresentar resultados distintos.

O fato de o modelo utilizado no estudo explicar em $48 \%$ a performance organizacional sugere que há outros fatores, além dos utilizados no estudo, que exercem influência sobre o desempenho das empresas. $\mathrm{O}$ acréscimo de novos construtos que, teoricamente, contribuam para a performance organizacional pode aumentar o poder de explicação do modelo.

A inserção de uma correlação entre o construto orientação para o mercado e performance organizacional, bem como daquela entre as variáveis que obtiveram correlações acima de 0,7 dentro do mesmo construto (OM2 <--> OM1; PO2 <--> PO1) consistem em modelos alternativos que podem ser testados em estudos futuros, a fim de verificar a sua influência sobre a performance das empresas. 
Com a realização do estudo, novas produto e processo, de que maneira elas questões de pesquisa podem ser contribuem para uma performance investigadas: qual a relação existente entre a organizacional superior - combinadas ou implementação da inovação de produto e individualmente? A situação econômica de processo nas empresas (positiva ou um país influencia a implementação das negativa)? Em se tratando das inovações de inovações de produto e processo? 


\section{REFERÊNCIAS}

APPOLINÁRIO, F. Metodologia da ciência: filosofia e prática da pesquisa. São Paulo: Pioneira Thomson Learning, 2006.

ARTZ, K. W.; NORMAN, P. M.; HATFIELD, D. E.; CARDINAL, L. B. A longitudinal study of the impact of $\mathrm{R} \& \mathrm{D}$, patents, and product innovation on firm performance. Journal of Product Innovation Management, v. 27, n. 5, p. 725-740, 2010.

ATUAHENE-GIMA, K. Market orientation and innovation. Journal of Business Research, v. 35, n. 2, p. 93-103, 1996.

ATUAHENE-GIMA, K.; KO, A. An empirical investigation of the effect of Market orientation and entrepreneurship orientation alignment on product innovation. Organization Science, v. 12, n. 1, p. 54,74, 2001.

BAKER, W. E.; SINKULA, J. M. Market orientation and the new product paradox. The Journal of Product Innovation Management, v. 22, n. 6, p. 483-502, 2005.

BLAUG, M. A survey of the theory of process-innovations. Economica, v. 30, n. 117, p. 13-32, New series, 1963.

BYRNE, B. M. Structural Equation Modeling with AMOS: basic concepts, applications, and programming. New York: Taylor \& Francis Group, 2010.

CARUNA, A.; PITT, L.; BERTHON, P. Excellence-market orientation link: some consequences for services firms. Journal of Business Research, v. 44, p. 5-15, 1998.

CHRISTENSEN, C. M.; BOWER, J. L. Customer power, strategic investment, and the failure of leading firms. Strategic Management Journal, v. 17, 197-218, 1996.

COOPER, R. G. Project newproduct: factors in new product success. European Journal of Marketing, v. 14, n. 5/6, p. 277-292, 1980.

COOPER, R. G. How new product strategies impact on performance. Journal of Product Innovation Management, v. 1, n. 1, p. 5-18, 1984.

DAMANPOUR, F. Organizational innovation: a meta-analysis of effects of determinants and moderators. Academy of Management Journal, v. 34, n. 3, p. 555-590, 1991. 
DAMANPOUR, F.; GOPALAKRISHNAN, S. The dynamics of the adoption of product and process innovation in organizations. Journal of Management Studies, v. 38, n. 1, p. 45-65, 2001.

DELANEY, J. T.; HUSELID, M. A. The impact of human resource management practice on perceptions of organizational performance. Academy of Management Journal, v. 39, n. 4, p. 949-969, 1996.

DOUGHERTY, D. A practice-centered model of organizational renewal through product innovation. Strategic Management Journal, v. 13, n. 1, p. 77-92, 1992. Special issue.

FORNELL, C.; LARCKER, D. Evaluating structural equation models with unobservable variables and measurements error. Journal of Marketing Research, v. 17, n. 1, 39-50, 1982.

GATIGNON, H.; XUEREB, J. Strategic orientation of the firm and new product performance. Journal of Marketing Research, v. 34, n. 1, p. 77-90, 1997. Special issue.

HAIR JR., J. F. ; BABIN, B.; MONEY, A. H.; SAMOUEL, P. Fundamentos de métodos de pesquisa em administração. Tradução Lene Belon Ribeiro. Porto Alegre: Bookman, 2005 .

HAIR JR., J. F. ; BLACK, W. C.; BARDIN, B. J.; ANDERSON, R. E. Multivariate Data Analysis. 7 ed., New Jersey: Prentice Hall, 2007.

HAIR JR., J. F.; BLACK, W. C.; BABIN, B. J.; ANDERSON, R. E.; TATHAM, R. L. Análise multivariada de dados. 6 ed., Porto Alegre: Bookman, 2009.

HAIR JR., J. F. ; BLACK, W. C.; BABIN, B. J.; ANDERSON, R. E. Multivariate data analysis: a global perspective. 7 ed. [S. 1.]: Pearson Prentice Hall, 2010.

HAN; J. K.; KIM, N.; SRIVASTAVA, R. K. Market orientation and organizational performance: is innovation the missing link? Journal of Marketing, v. 62, n. 4, p. 3045, 1998.

HULT, G. T. M.; HURLEY, R. F.; KNIGHT, G. A. Inovativeness: its antecedents and impact on business performance. Industrial Marketing Management, v. 33, p. 429-438, 2004. 
HURLEY; R. F.; HULT, G. T. M. Innovation, market orientation, and organizational learning: an integration and empirical examination. Journal of Marketing, v. 62, p. 42$54,1998$.

KIM, W. C.; MAUBOURGNE, R. Blue ocean strategy. Harvard Business Review, p. $1-9,2004$.

KLINE, R. B. Principles and practice of structural equation modeling. 2 ed. New York: The Guilford Press. 2005.

KLINE, R. B. Principle and practice of structural equation modeling. 3 ed. New York London: The Guilford Press, 2011.

KOHLI, A. K.; JAWORSKI, B. J. Market orientation: the construct, research propositions, and managerial implications. Journal of Marketing, v. 54, n. 2, p. 1-18, 1990.

KOHLI, A. K.; JAWORSKI, B. J. Market orientation: antecedents and consequences. Journal of Marketing, v. 57, n. 3, p. 53-70, 1993.

LAWTON, L.; PARASURAMAN, A. The impact of the marketing concept on new product planning. Journal of Marketing, v. 44, n. 1, p. 19-25, 1980.

LI, C. LIN, C.; CHU, C. The nature of market orientation and the ambidexterity of innovations. Management Decision, v. 46, n. 7, p. 1002-1026, 2008.

LI, H.; ATUAHENE-GIMA, K. Product inovation strategy and the performance of new technology ventures in China. Academy of Management Journal, v. 44, n. 6, p. 1123 1134, 2001.

LIN, R.; TAM, K.; GENG, Y. Market demand, green product innovation, and firm performance: evidence from Vietnam motorcycle industry. Journal of Cleaner Production, v. 40, p. 101-107, 2013.

LIU, J.; SU, J. How does market orientation affect product innovation in China's manufacturing industry: the contingent value of dynamic capabilities. In: INTERNATIONAL CONFERENCE ON EDUCATION TECHNOLOGY AND MANAGEMENT SCIENCE (ICETMS), 2012, Nanjing. Proceedings..., Atlantis Press, 2013, p. 165-169. 
LUKAS, B. A.; FERREL, O. C. The effect of market orientation on product innovation. Journal of the Academy of Marketing Science, v. 28, n. 2, p. 239-247, 2000.

MALHOTRA, N. K. Pesquisa de marketing: foco na decisão. Trad. Opportunity Translations. Rev. técn. Maria Cecília Laudísio e Guilherme de Farias Shiraishi. $3^{\text {a }}$ ed. São Paulo: Pearson Prentice Hall, 2011.

MALHOTRA, N. K. Pesquisa de marketing: uma orientação aplicada. Bookman Editora, 2012.

MARCH, J. G.; SUTTON, R. I. Organizational performance as a dependent variable. Organization Science, v. 8, n. 6, p. 698-706, 1997.

MARDIA, K.V. Measures of multivariate skewness and kurtosis with applications. Biometrika, v. 36, p. 519-530, 1970.

MAROCO, J. Análise de equações estruturais: fundamentos teóricos, softwares \& aplicações. PSE, Lisboa, 2010.

MELVILLE, N.; KRAEMER, K.; GURBAXANI, V. Review: Information technology and organizational performance: an integrative model of it business value. MIS Quarterly, v. 28, n. 2, p. 283-322, 2004.

MITCHELL, W. Are more good things better, or will technical and market capabilities conflict when a firm expands? Industrial and Corporate Change, v. 1, n. 2, p. 327-346, 1992.

NARVER, J. C.; SLATER, S. F. The effect of a market orientation on business profitability. Journal of Marketing, v. 54, n. 4, p. 20-35, 1990.

NARVER, J. C.; SLATER, S. F.; TIETJE, B. Creating a market orientation. Journal of Market-Focused Management, v. 2, n. 3, p. 241-255, 1998.

OCDE - ORGANIZAÇÃO PARA A COOPERAÇÃO E DESENVOLVIMENTO ECONÔMICO. Manual de Oslo: diretrizes para coleta e interpretação de dados sobre inovação. 3 ed. [S. 1.], 2005.

PALADINO, A. Investigating the drivers of innovation and new product success: a comparison of strategic orientations. The Journal of Product Innovation Management, v. 24, n. 6, p. 534-553, 2007. 
PESTANA, M. H., GAGEIRO, J.N. Análise de dados para ciências sociais: a complementariedade do SPSS, $4^{\text {a }}$ ed. Sílabo, Lisboa, 2005.

PORTER, M. Capital disadvantage: America's failing capital investment system. Harvard Business Review, n. 70, p. 65-82, 1992.

POWELL, T. C. Organizational alignment as competitive advantage. Strategic Management Journal, v. 13, p. 119-134, 1992.

REINARTZ, W.; KRAFFT, M.; HOYER, W. D. The customer relationship management process: its measurement and impact on performance. Journal of Marketing Research, v. 41, n. 3, p. 293-305, 2004.

ROESCH, S. M. A. Projetos de estágio e de pesquisa em administração: guia para estágios, trabalhos de conclusão, dissertações e estudos de caso. Col. Grace Vieira Becker, Maria Ivone de Mello. $3^{\text {a }}$ ed. $8^{\text {a }}$ reimp. São Paulo: Atlas, 2013.

ROSENBUSCH, N.; BRINCKMANN, J.; BAUSCH, A. Is innovation always beneficial? A meta-analysis of the relationship between innovation and performance in SMEs. Journal of Business Venturing, v. 26, n. 4, p. 441-457, 2010.

SALVADÓ-AMORES, J.; CASTRO, G. M.; NAVAS-LÓPEZ, J. E. Green corporate image: moderating the connection between environmental product innovation and firm performance. Journal of Cleaner Production, v. 83, p. 356-365, 2014.

SCHROEDER, D. M. A dynamic perspective on the impact of process innovation upon competitive strategies. Strategic Management Journal, v. 11, n. 1, p. 25-41, 1990.

SCHUMPETER, J. A. The theory of economic development: an inquiry into profits, capital, credit, interest, and the business cycle. Cambridge: Harvard University Press, 1934.

SLATER, S. F.; NARVER, J. C. Does competitive environment moderate the market orientation-performance relationship? Journal of Marketing, v. 58, n. 1, p. 46-55, 1994.

SLATER, S. F.; NARVER, J. C. Market orientation, customer value, and superior performance. Business Horizons, v. 37, n. 2, p. 22-28, 1994. 
SLATER, S. F.; NARVER, J. C. Market orientation and the learning organization. Journal of Marketing, v. 59, n. 3, p. 63,74, 1995.

SOK, P.; O'CASS, A. Examining the new product innovation-performance relationship: optimizing the role of individual-level creativity and attention-to-detail. Industrial Marketing Management, v. 47, p. 156-165, 2015.

TANAKA, J. S. Multifaceted conceptions on fit in structural equations modeling. In: BOLLEN, K. A.; LONG, J. S. (Ed). Testing structural equation models. Newbury Park: Sage, p. 10-39, 1993.

VERHEES, F. J. H. M.; MEULENBERG, M. T. G. Market orientation, innovativeness, product innovation, and performance in small firms. Journal of Small Business Management, v. 42, n. 2, p. 134-154, 2004. 